\title{
Advance online publication
}

Nature Immunology is going weekly!

Actually, we are still a monthly print journal, but we now publish manuscripts online weekly. It is the goal of the Nature Publishing Group to roll out this service for all of the Nature Research Journals in the next few months. We feel that this provides an important service for our authors in a field as competitive as immunology. Our readers also benefit, as they now have access to papers weeks earlier than with our monthly system. The papers are available in our new Advance Online Publication (AOP) section of the Nature Immunology website (just click on the AOP icon in the left-hand navigation bar). Unlike some journals, what we post is the final version of the paper, which is complete except for page numbers. When the regular monthly issue goes live on our website, we move those papers that were in the AOP section to the Table of Contents and add the page numbers, so that the online version matches the paper as printed. We are currently publishing only our research papers in this fashion, but are considering strategies that would make it possible to publish even more of the journal, such as News \& Views, by AOP.

How does it work? When a paper is accepted, we copyedit it and create a page proof for authors. Once author corrections come in, the paper is scheduled for AOP. We will be notifying authors of the publication date so that their institutes can coordinate any press releases or other publicity without breaking the news embargo on the paper (announcements or news items about all papers are embargoed until 2 p.m. Eastern Time the day before publication). Papers with AOP will have PDFs that clearly indicate that they are AOPs at the bottom of the page.

Papers that are published online before being compiled into a monthly issue will not have their final page or volume numbers. Instead citation is done by utilizing the Digital Object Identifier (DOI) code that will be published with each paper and displayed in the PDF version. DOIs are labels, provided by the nonprofit International DOI Foundation, that can be used for citation, in lieu of volume and pages, and for viewing AOP papers. The format for citing a paper should include the phrase, "Nature Immunology, <date>, DOI:<paper's DOI>". The system for linking published papers to their DOIs is maintained by
CrossRef, another nonprofit organization, of which the Nature Publishing Group is a founding member.

Here is an example of DOIs in action. A reader of a J. Exp. Med. paper comes upon a citation that directs the reader to a Nature Immunology paper with a DOI, perhaps because at the time the J. Exp. Med. paper was printed, the Nature Immunology paper was only available online. To find the paper, one could search the Nature Immunology website for the paper by author, as you can't assume that the paper is still in the AOP section by the time the reader encounters the citation. A more direct route would be to type the URL http://dx.doi.org/<DOIofpaper $\geq$ (for instance, the URL http://dx.doi.org/10.1038/ni713 will take you to a paper in this month's issue) into a browser. All Nature Immunology DOIs start with 10.1038/ni and then have a random number identifier. The DOI will link to a Nature Immunology web page that contains the abstract and access to the full paper and PDF.

One can tell if a Nature Immunology paper has been published in print or not by looking at the PDF. All Nature Immunology papers that are still in the AOP section have the words "advance online publication" at the bottom of the page (pages in all AOP papers are numbered from 1 to $n$, but these do not correspond to the page numbers assigned when printed). After a paper is published in print, the DOI will still workthat is, it continues to link to the same paper-but now the PDF will have the actual volume and page numbers at the bottom. The beauty of the DOI for publishers is that the identifier will always travel with the paper, even if web addresses change or PDFs are updated.

Online publication should be considered public disclosure, although how this is viewed by the courts, indexing services and Medline is still an evolving issue. Our responsibility as a journal is to make clear when the information was presented to the public: the first page of each Nature Immunology research paper now shows the date that the paper was published online. A paper published in print in January 2002, but published online in December 2001 is still considered a 2002 paper by most publishers and Medline, but as more journals adopt advance online publication we may see the primacy of print seriously challenged. 